\title{
The Efficacy of Simultaneous Breast Reconstruction and Contralateral Balancing Procedures in Reducing the Need for Second Stage Operations
}

\author{
Mark L Smith ${ }^{1}$, Emily M Clarke-Pearson ${ }^{1}$, Michael Vornovitsky ${ }^{1}$, Joseph H Dayan ${ }^{1}$, \\ William Samson ${ }^{2}$, Mark R Sultan ${ }^{2}$ \\ ${ }^{I}$ Department of Surgery, Mount Sinai Beth Israel, New York, NY; ${ }^{2}$ Department of Surgery, Mount Sinai St. Luke's-Roosevelt, New York, NY, \\ USA
}

Background Patients having unilateral breast reconstruction often require a second stage procedure on the contralateral breast to improve symmetry. In order to provide immediate symmetry and minimize the frequency and extent of secondary procedures, we began performing simultaneous contralateral balancing operations at the time of initial reconstruction. This study examines the indications, safety, and efficacy of this approach.

Methods One-hundred and two consecutive breast reconstructions with simultaneous contralateral balancing procedures were identified. Data included patient age, body mass index (BMI), type of reconstruction and balancing procedure, specimen weight, transfusion requirement, complications and additional surgery under anesthesia. Unpaired t-tests were used to compare BMI, specimen weight and need for non-autologous transfusion.

Results Average patient age was 48 years. The majority had autologous tissue-only reconstructions (94\%) and the rest prosthesis-based reconstructions (6\%). Balancing procedures included reduction mammoplasty (50\%), mastopexy (49\%), and augmentation mammoplasty (1\%). Average BMI was 27 and average reduction specimen was 340 grams. Non-autologous blood transfusion rate was 9\%. There was no relationship between BMI or reduction specimen weight and need for transfusion. We performed secondary surgery in $24 \%$ of the autologous group and $100 \%$ of the prosthesis group. Revision rate for symmetry was 13\% in the autologous group and $17 \%$ in the prosthesis group.

Conclusions Performing balancing at the time of breast reconstruction is safe and most effective in autologous reconstructions, where $87 \%$ did not require a second operation for symmetry.

Keywords Breast neoplasms / Mammoplasty / Free tissue flaps
Correspondence: Mark L Smith Department of Surgery, Mount Sinai Beth Israel, 10 Union Square East,

Suite 2L, New York,

NY 10003, USA

Tel: +1-212-844-8796

Fax: +1-212-844-8798

E-mail: msmith@chpnet.org
This article was presented at the Chang Gung-Mayo Clinic Symposium in Reconstructive Surgery on October 2011 in Taipei, Taiwan.

No potential conflict of interest relevant to this article was reported.

\section{INTRODUCTION}

The goal of breast reconstruction in women undergoing mas- tectomy includes a cosmetically acceptable reconstructed breast in addition to symmetry with the contralateral breast. Clinical series show that as many as $86 \%$ of patients undergoing unilat- 
eral reconstruction benefit from a contralateral breast procedure to improve symmetry [1-6]. Many surgeons opt to delay contralateral breast procedures for a planned second surgery, where further shaping of the reconstructed breast may also be performed. However, one of the presumed benefits of autologous reconstruction over expander/implant-based reconstruction is the opportunity to avoid undergoing a second surgery, anesthesia, and recuperation. A planned second-stage procedure effectively eliminates that benefit [5]. As part of an overall approach to provide immediate symmetry and to minimize the number of secondary procedures, as well as their scope, we began shaping both breasts as much as possible at the initial operation. In addition to the contralateral balancing procedure, we performed extensive suture suspension and sculpting of the autologous reconstructed breast and paid close attention to the donor site closure at the initial surgery to optimize the patients' appearance at the first operation.

The potential advantages of this single-stage approach include: reduced number of surgeries, decreased recuperation, decreased expense, and avoidance of an extended interval of asymmetry, especially if the secondary procedure is postponed by adjuvant therapy. In addition, if revisions are required, they may be less extensive and more precise.

Concerns that are often raised regarding reconstruction and simultaneous contralateral balancing are that added surgery and operative time may increase complications or necessitate blood transfusions, and changes may occur in breast shape during healing that lead to new asymmetries. We reviewed our experience with simultaneous balancing procedures to examine the validity of these concerns.

\section{METHODS}

Institutional review board approval was obtained for this study. All available records for breast reconstruction performed by the senior authors (M.L.S. and M.R.S.) between 1999 and 2006 were reviewed and 102 breast reconstructions with simultaneous contralateral balancing procedures were identified. Data obtained included: patient age, body mass index (BMI), type of reconstruction and balancing procedure, specimen weight, transfusion requirement, major and minor complications and revision surgery.

We categorized balancing procedure as a reduction mammoplasty or mastopexy based on specimen size, with greater than $100 \mathrm{~g}$ being considered a reduction mammoplasty and $100 \mathrm{~g}$ or less being considered a mastopexy. We selected $100 \mathrm{~g}$ as the cutoff because we felt it represented a noticeable size difference between breasts. Therefore, procedures with specimens larger than
$100 \mathrm{~g}$ were done to address volume discrepancies, while specimens of $100 \mathrm{~g}$ or less were felt to primarily address ptosis. We included augmentation mammoplasty as a separate category.

Transfusion requirement was analyzed using unpaired t-tests to assess for any correlation between BMI, specimen weight and need for non-autologous transfusion.

Complications were considered major if they extended the hospital stay or required readmission to the hospital or return to the operating room within 30 days of surgery. Complications were considered minor if they did not extend hospital stay or if readmission to the hospital or return to operating room (OR) occurred more than 30 days after the initial surgery. We examined revision surgery by reconstruction type (autologous vs. prosthetic), whether or not it was done to improve symmetry, and whether the reconstructed or contralateral breast was revised. Rates of revision surgery between the two groups were statistically compared using a two-tailed Fisher's exact test.

\section{RESULTS}

\section{Procedures performed}

The average patient age was 48 years. The majority of reconstructions were autologous tissue-only (94\%) and consisted of pedicled transverse rectus abdominis myocutaneous (TRAM) flaps (54), deep inferior epigastric perforator (DIEP) or muscle-sparing free TRAM flaps (41), and a latissimus flap (1). The remaining reconstructions were prosthesis-based $(6 \%)$ and consisted of latissimus flaps over either an expander (2) or implant (1), or tissue expanders without flap coverage (3). Balancing procedures consisted of reduction mammoplasty (51), mastopexy (50), and augmentation mammoplasty (1). Minimum follow-up was 12 months. A representative patient is shown in Figs. 1, 2.

\section{BMI, specimen weight, and transfusion requirement} The average BMI was $27 \mathrm{~kg} / \mathrm{m}^{2}$ (range, $15-42 \mathrm{~kg} / \mathrm{m}^{2}$ ) and the average reduction specimen (with $101 \mathrm{~g}$ being the minimum to be considered a reduction mammoplasty) was $340 \mathrm{~g}$ (range, $101-1,160 \mathrm{~g})$. Nine (9\%) patients received non-autologous blood transfusion. There was no relationship between BMI or specimen weight and the need for transfusion.

\section{Complications}

The overall complication rate was $11 \%$, with one major medical, four major surgical and seven minor surgical complications. The major medical complication was a deep vein thrombosis requiring readmission to the hospital. The four major surgical complications occurred in three patients. One patient had a hematoma in the contralateral breast requiring return to $\mathrm{OR}$ on postopera- 


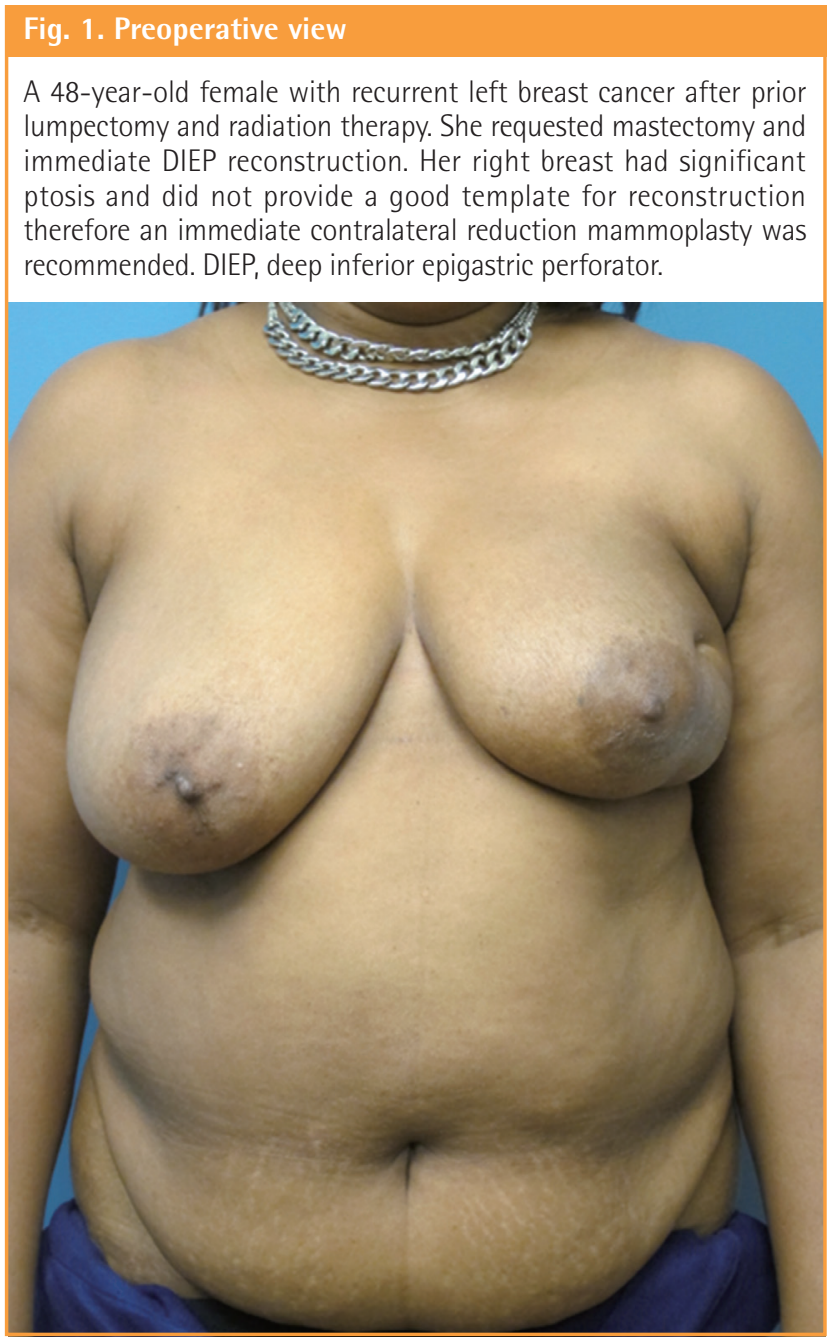

tive day two and then a fever on postoperative day eight requiring readmission for antibiotic therapy (these were counted as two major complications). The second patient had a hematoma at the site of her sentinel node biopsy that required immediate return to the operating room for evacuation. The third patient had epidermolysis of her mastectomy flaps requiring early revision.

Six complications were considered minor, and consisted primarily of small areas of fat necrosis in the flap or minor mastectomy flap epidermolysis.

\section{Revision surgery}

Overall, 29 of 102 (28\%) patients required additional surgery to address symmetry, complications, scars or the donor site. Nipple reconstructions were typically done under local anesthesia in an office setting and were not included as revision surgery.

\section{Autologous reconstruction group}

$76 \%$ of patients undergoing autologous reconstruction required no additional surgery. 23 of the 96 autologous tissue-only pa-

\section{Fig. 2. Postoperative view}

Same patient after left DIEP reconstruction and simultaneous contralateral reduction (170 g removed). The patient was satisfied with the result and desired no further revisions. DIEP, deep inferior epigastric perforator.

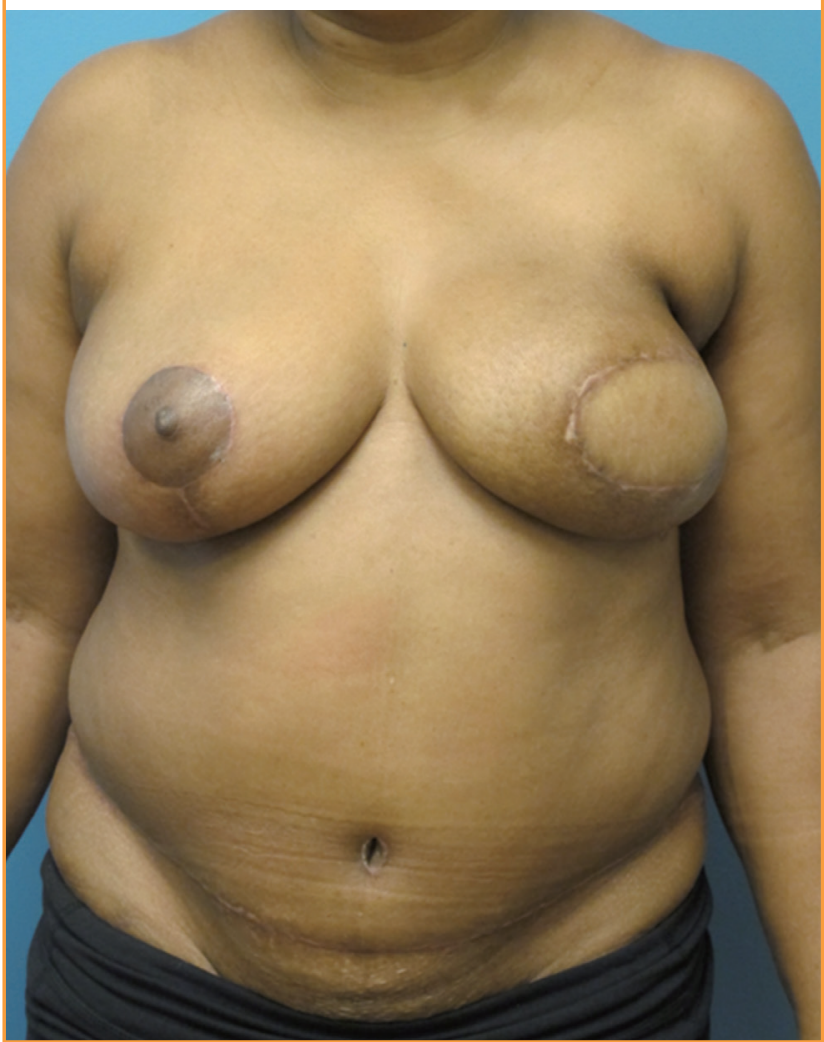

tients (24\%) required a second procedure, 12 (13\%) for symmetry and $11(12 \%)$ due to reasons unrelated to symmetry. Of the patients undergoing revisions for symmetry, 8 had surgery on the reconstructed breast, 1 on the contralateral breast, and 3 on both breasts.

\section{Prosthesis-based reconstruction group}

$100 \%$ of patients with prosthesis-based reconstruction required secondary surgery to either remove an expander (1), exchange an expander for an implant (4) or to replace an implant (1). This was a significantly greater percentage than in the autologous group $(\mathrm{P}<0.0004)$. Only one patient $(17 \%)$ required revision for symmetry. A reduction was performed on her contralateral breast after a 25-pound weight loss.

\section{DISCUSSION}

\section{Safety and efficacy}

Many surgeons prefer to perform balancing procedures at a second-stage operation because it is believed that it is safer and better symmetry will be attained, however there is little data to sup- 
port this assertion. In patients who are left with a pronounced asymmetry at their initial procedure, there is little choice but to undergo a secondary procedure if they want to properly fit into their bra or clothes. The goal of performing the balancing procedure at the time of the initial reconstruction is provide immediate breast symmetry and avoid the need for secondary procedures while maintaining patient safety. Overall, the complication rate $(11 \%)$ and transfusion rate $(9 \%)$ in this study were low. The complication rate for the contralateral breast was extremely low (1\%). Although previous studies have shown that neither breast reductions nor autologous breast reconstructions are independently associated with a high rate of blood transfusion, a concern in performing them simultaneously is that the blood loss for the combined procedures may cause an unacceptably high rate of transfusion [7-11]. Obese patients, those requiring larger reductions, and patients undergoing autologous reconstruction are theoretically prone to increased blood loss due to more extensive dissection. Nineteen percent of the patients in the study had a BMI greater than 30 and were considered obese. BMI and balancing reduction specimen weight did correlate; however, there was no correlation between BMI or specimen weight and the need for transfusion. The 9\% transfusion rate in this study compares favorably to a recent paper by Appleton et al. [12] reporting an $11 \%$ transfusion rate for unilateral DIEP flaps.

In the autologous reconstruction group, $24 \%$ of patients returned to the OR for additional surgery either for breast contouring or to address a complication. Since all patients in this study were deemed as requiring a contralateral procedure for symmetry, performing a simultaneous balancing procedure at the time of initial reconstruction resulted in a $76 \%$ reduction in patients requiring additional surgery of any kind. Only $13 \%$ of patients had secondary procedures for symmetry, leaving $87 \%$ of patients who did not require additional surgery for symmetry. In the prosthetic reconstruction group, all six patients required a secondary operation either to exchange a tissue expander for an implant, or to replace an implant. This group was small and varied with three patients having latissimus flaps with either an expander or implant and three having a tissue expander alone for their initial reconstructive procedure. Simultaneous balancing procedures were done in these patients primarily to minimize asymmetry during the interval between expander placement and implant exchange and as a guide to tissue expansion. This limited sample size lacked statistical power from which to draw a conclusion; however there were no transfusions and no major complications attributable to the contralateral balancing procedure in this group.

\section{Indications and approach}

We consider immediate contralateral balancing in patients who clearly will require a contralateral procedure to achieve symmetry with the reconstructed breast. This commonly occurs in patients with large or ptotic breasts, where the patient could benefit from a reduction or mastopexy. Also, it is helpful when there is inadequate donor site tissue to match the contralateral breast volume or ptosis, or when there is a significant skin deficit, as may occur in delayed reconstructions. By performing the balancing procedure at the initial operation, the surgeon can see the shape and volume of the contralateral breast and use it as a template for the reconstruction. This avoids the temptation to leave additional flap tissue to match a larger, non-adjusted contralateral breast. In patients who are actively smoking or where it is unclear if a contralateral reduction or mastopexy will ultimately be needed, we would delay the balancing procedure until a second stage.

A key component of single stage autologous reconstruction with contralateral balancing is shaping the flap as precisely as possible in the primary setting. The shaping is facilitated by maximal exposure to the flap before it is fully inset, which is difficult to achieve in the secondary setting. The contralateral breast is usually modified prior to shaping of the reconstructed breast so it may serve as a guide to the reconstruction. The mastopexy or reduction will often have some degree of settling, but the dermal elements and superficial fascia in a flap provide support that limit settling of the reconstructed breast. It is recommended to take this into consideration during shaping and to slightly overcorrect the contralateral breast and create slightly more ptosis in the reconstructed breast. Care must also be taken not to create too much projection in the contralateral breast, as this may be difficult to match in autologous reconstruction.

\section{Logistics}

The contralateral balancing procedure takes approximately 20 30 minutes to excise and staple closed and is performed during the mastectomy in immediate autologous reconstructions. The surgical assistant then replaces the staples with sutures while the flap harvest commences, thus minimizing additional operative time.

\section{Review of literature}

Several large studies characterize the patient population requiring contralateral balancing procedures. Both Nahabedian $[1,2]$ and Losken et al. [3] found that patients who undergo delayed breast reconstruction require a contralateral balancing procedure more often than patients undergoing immediate reconstruction. Rates of contralateral balancing procedures ranged 
from $13 \%-46 \%$ in immediate reconstructions to $24 \%-86 \%$ in delayed reconstructions, with variation attributed to the type of reconstruction. In our series, only six patients had delayed reconstructions, as the majority of patients treated at our institution are seen for immediate reconstruction after mastectomy. We did not compare simultaneous balancing procedures between the immediate and delayed group, as the number of delayed reconstructions was not large enough to reflect meaningful trends in management.

Few studies address the timing of balancing procedures in relation to breast reconstruction. In Nahabedian's and Losken's series, all patients underwent balancing procedures at a second stage. Enajat et al. [5] point out that while patients are routinely informed that expander/implant reconstruction is at least a twostage procedure, patients undergoing autologous reconstruction are often under the impression that if all goes as planned, they will only require a single operation to reconstruct the breast mound. They point out that a large number of patients undergoing autologous reconstruction require secondary revisions to the reconstructed breast for shaping, the contralateral breast for symmetry and the donor site.

A number of smaller studies allude to simultaneous breast reconstruction and contralateral procedures, but do not comment on the indications, safety or approach [6,13-19]. Hudson and Skoll [18] described using a simultaneous contralateral balancing procedure in a series of 18 patients with large breasts undergoing single-stage breast reconstruction using an implant. They reported no complications in the contralateral breast, but their mean follow-up was less than one year and despite mentioning surgical complications in the reconstructed breast, they did not discuss revisions. Losken et al. [19] compared 51 patients undergoing partial mastectomy using an oncoplastic reduction mammaplasty technique and a contralateral reduction for symmetry, with 30 patients who had a skin-sparing mastectomy and contralateral breast reduction. Although many of the partial breast reconstruction patients had simultaneous contralateral reductions, only a small percentage of total breast reconstruction patients had a simultaneous reduction. The paper did not discuss complications or outcomes in regards to the contralateral breast, however there were significantly more revisions in the breast reconstruction group. Stevenson and Goldstein [20] did a retrospective review of 25 patients who underwent pedicled TRAM reconstruction with simultaneous balancing procedures compared to 14 who had TRAM reconstruction alone. They found no differences in operating room time, blood loss, or length of hospital stay between the two groups; and no patients required revision of the contralateral side for symmetry. Their average follow-up was 16 months. Huang et al. [21] also published a recent report on 22 abdominal free flap unilateral breast reconstruction with simultaneous balancing procedures and noted that cosmesis was better when compared to patients undergoing similar reconstruction alone. More recently, Inbal et al. [22] compared a consecutive series of DIEP flap patients where 33 patients had immediate contralateral balancing procedures, 8 had secondary contralateral balancing procedures, and 10 did not require a contralateral balancing procedures. They found no difference in revisions to the reconstructed breast between the three groups and no differences in revisions in the contralateral balanced breast between the immediate and delayed contralateral balancing groups. Quality of life at the completion of reconstruction was similar in all three groups, however there was a significant decrease in quality of life in the delayed balancing procedure group during the period between the breast reconstruction and performance of the balancing procedure, demonstrating that importance of providing immediate symmetry [22].

In the only other large series of patients undergoing free flap breast reconstruction and immediate contralateral procedures for symmetry, Chang et al. [23] found that $50 \%$ of patients undergoing breast reconstruction ultimately require a contralateral balancing procedure. In their series, 14\% (154 of 1,120) of patients underwent an immediate contralateral procedure for symmetry and $36 \%(404$ of 1,120$)$ of patients underwent a delayed contralateral procedure. Revision of the contralateral symmetry procedures was performed in $21 \%$ of patients, overall. The revision rate was higher for augmentations and mastopexies in the immediate group compared to the delayed group but there was no difference in revision rates for breast reductions between groups. They found a higher incidence of complications in the immediate balancing procedure group but that the average number of procedures performed was significantly higher in those undergoing a delayed contralateral procedure compared to an immediate contralateral procedure [23]. Our study provides further supports of these findings by demonstrating a significant reduction in secondary procedures (24\%) and revisions for symmetry $(13 \%)$ in patients undergoing autologous reconstruction and immediate contralateral procedures for symmetry.

Patients undergoing tissue expansion and implant reconstruction are even more likely to require a balancing procedure. Two series show that $62 \%-66 \%$ of women undergoing expander/ implant reconstruction require contralateral balancing, compared to $37 \%-41 \%$ of autologous reconstructions $[3,24]$. Optimal timing for the balancing procedure in tissue-expander based reconstruction is still unclear. Our study had only six patients with tissue-expanders and therefore no meaningful conclusion could be drawn. 


\section{Limitations}

This study is limited by its retrospective nature and lack of a matched control group. In addition, an assumption was made that patients who did not have revisions achieved acceptable symmetry. Even in the best reconstructions, perfect symmetry is seldom achieved and most patients have a degree of asymmetry they are willing to accept. Therefore, we used revision rate for symmetry as an indirect measure of unacceptable asymmetry [25].

\section{Conclusions}

In patients undergoing unilateral autologous reconstruction who required contralateral procedures for symmetry, $87 \%$ of patients did not require a second surgery when immediate contralateral balancing was performed. Performing balancing procedures at the time of a unilateral breast reconstruction is associated with a low rate of complication, transfusion and revision and therefore may be preferable to staged procedures in properly selected patients. It remains unclear when the optimal time is to perform a contralateral balancing procedure in patients undergoing prosthetic implant-based reconstruction.

\section{REFERENCES}

1. Nahabedian MY. Symmetrical breast reconstruction: analysis of secondary procedures after reconstruction with implants and autologous tissue. Plast Reconstr Surg 2005;115: 257-60.

2. Nahabedian MY. Managing the opposite breast: contralateral symmetry procedures. Cancer J 2008;14:258-63.

3. Losken A, Carlson GW, Bostwick J 3rd, et al. Trends in unilateral breast reconstruction and management of the contralateral breast: the Emory experience. Plast Reconstr Surg 2002;110:89-97.

4. Asplund O, Svane G. Adjustment of the contralateral breast following breast reconstruction. Scand J Plast Reconstr Surg 1983;17:225-32.

5. Enajat M, Smit JM, Rozen WM, et al. Aesthetic refinements and reoperative procedures following 370 consecutive DIEP and SIEA flap breast reconstructions: important considerations for patient consent. Aesthetic Plast Surg 2010; 34:306-12.

6. Panettiere P, Marchetti L, Accorsi D, et al. Aesthetic breast reconstruction. Aesthetic Plast Surg 2002;26:429-35.

7. Tzilinis A, Lofman AM, Tzarnas CD. Transfusion requirements for TRAM flap postmastectomy breast reconstruction. Ann Plast Surg 2003;50:623-7.

8. Lennox PA, Clugston PA, Beasley ME, et al. Autologous blood transfusion in TRAM breast reconstruction: is it necessary? Ann Plast Surg 2004;53:532-5.

9. Cohen J. Is blood transfusion necessary in reduction mammaplasty patients? Ann Plast Surg 1996;37:116-8.

10. Clugston PA, Fitzpatrick DG, Kester DA, et al. Autologous blood use in reduction mammaplasty: is it justified? Plast Reconstr Surg 1995;95:824-8.

11. Buenaventura S, Severinac R, Mullis W, et al. Outpatient reduction mammaplasty: a review of 338 consecutive cases. Ann Plast Surg 1996;36:162-6.

12. Appleton SE, Ngan A, Kent B, et al. Risk factors influencing transfusion rates in DIEP flap breast reconstruction. Plast Reconstr Surg 2011;127:1773-82.

13. Tran NV, Evans GR, Kroll SS, et al. Postoperative adjuvant irradiation: effects on tranverse rectus abdominis muscle flap breast reconstruction. Plast Reconstr Surg 2000;106: 313-7.

14. Smith ML, Evans GR, Gurlek A, et al. Reduction mammaplasty: its role in breast conservation surgery for early-stage breast cancer. Ann Plast Surg 1998;41:234-9.

15. Petit J, Rietjens M, Garusi C. Breast reconstructive techniques in cancer patients: which ones, when to apply, which immediate and long term risks? Crit Rev Oncol Hematol 2001;38:231-9.

16. Venus MR, Prinsloo DJ. Immediate breast reconstruction with latissimus dorsi flap and implant: audit of outcomes and patient satisfaction survey.J Plast Reconstr Aesthet Surg 2010;63:101-5.

17. Song HM, Styblo TM, Carlson GW, et al. The use of oncoplastic reduction techniques to reconstruct partial mastectomy defects in women with ductal carcinoma in situ. Breast J 2010;16:141-6.

18. Hudson DA, Skoll PJ. Complete one-stage, immediate breast reconstruction with prosthetic material in patients with large or ptotic breasts. Plast Reconstr Surg 2002;110: 487-93.

19. Losken A, Pinell XA, Eskenazi B. The benefits of partial versus total breast reconstruction for women with macromastia. Plast Reconstr Surg 2010;125:1051-6.

20. Stevenson TR, Goldstein JA. TRAM flap breast reconstruction and contralateral reduction or mastopexy. Plast Reconstr Surg 1993;92:228-33.

21. Huang JJ, Wu CW, Leon Lam W, et al. Simultaneous contralateral breast reduction/mastopexy with unilateral breast reconstruction using free abdominal flaps. Ann Plast Surg 2011;67:336-42.

22. Inbal A, Gur E, Otremski E, et al. Simultaneous contralateral breast adjustment in unilateral deep inferior epigastric per- 
forator breast reconstruction. J Reconstr Microsurg 2012; 28:285-92.

23. Chang EI, Selber JC, Chang EI, et al. Choosing the optimal timing for contralateral symmetry procedures after unilateral free flap breast reconstruction. Ann Plast Surg 2013 Jun 12 [Epub]. http://dx.doi.org./0.1097/SAP.0b013e31828bble3.

24. Giacalone PL, Bricout N, Dantas MJ, et al. Achieving sym- metry in unilateral breast reconstruction: 17 years experience with 683 patients. Aesthetic Plast Surg 2002;26:299302.

25. Gui GP, Kadayaprath G, Tan SM, et al. Evaluation of outcome after immediate breast reconstruction: prospective comparison of four methods. Plast Reconstr Surg 2005; 115:1916-26. 